\title{
Estudio de impacto social de los colegios del Sistema Educativo Arquidiocesano de Bogotá-SEAB de la Localidad Rafael Uribe (2016-2017) ${ }^{1}$ Study of the social impact of SEAB - Archdiocesan Educational System of Bogo- tá schools in Locality Rafael Uribe (2016-2017)
}

\author{
Patricia Eugenia Carrera Díaz ${ }^{2}$ \\ Luzmar Durán Vega ${ }^{3}$
}

Resumen

Este artículo responde al Estudio de Impacto Social de los Colegios del Sistema Educativo de la Arquidiócesis de Bogotá-SEAB de la Localidad Rafael Uribe Uribe, realizado entre el 2016 y el 2017, el cual incluyó 7 colegios. Se tuvo en cuenta la comunidad académica (directivos, administrativos, docentes, estudiantes, padres de familia, egresados), así como las comunidades donde se encuentran ubicadas estas instituciones. El tipo de investigación es de impacto no recurrente y sigue un enfoque mixto. Se realizaron entrevistas abiertas y semiestructuradas que se triangularon con encuestas. Se encuentra que, a través de la filosofía cristiana católica, las parroquias e instituciones educativas se articulan, observando similitudes, tensiones y especificidades institucionales en relación con los territorios y poblaciones beneficiarias de la localidad, según su configuración e inciden en el nivel de impacto interno y en su contexto, aportando al capital humano y social. Se propone potenciar el sistema al cual se adhieren para visibilizar dicho impacto, fortaleciendo las relaciones con instituciones que hacen parte del Sistema Educativo de la Arquidiócesis de Bogotá, la comunidad y sus egresados.

Palabras claves: Educación básica y media; Impacto social; Colegio parroquial; Sistema Educativo de la Arquidiócesis de Bogotá - SEAB.

\begin{abstract}
This article presents a study of the Social Impact of SEAB (Archdiocesan Educational System of Bogotá) schools in the locality Rafael Uribe Uribe, carried out between 2016 and 2017, which included 7 schools. The academic community was taken into account (managers, administrators, teachers, students, parents, graduates), as well as the communities where these institutions are located. The type of research corresponds to one of non-recurrent impact, following a mixed approach. Open and semi-structured interviews were conducted and triangulated with surveys. It was found that, through Catholic Christian philosophy, the parishes and educational institutions are articulated, observing similarities, tensions and institutional specificities related to the beneficiary territories and populations of the locality according to their configuration. It affects the level of internal impact and its context, contributing to human and social capital. It is proposed to strengthen the system to which these institutions are part to make this impact visible, strengthening relations with the institutions that are part of the Educational System of the Archdiocese of Bogotá, the community and its graduates.
\end{abstract}

Keywords: Elementary and high school education; Social impact; Parish school; Educational System of the Archdiocese of Bogotá - SEAB.

\footnotetext{
${ }^{1}$ Artículo de investigación.

${ }^{2}$ Mg. Sociología. Secretaría Distrital de Gobierno - Dirección de Derechos Humanos. https://orcid.org/0000-0003-4269-2095 E-mail: pcarreradiaz@gmail.com

${ }^{3} \mathrm{Mg}$. Educación. https://orcid.org/0000-0001-6186-1371 E-mail: luduves@gmail.com

*Cómo citar este artículo: Carrera, P., y Durán, L. (2018). Estudio de impacto social de los colegios del Sistema Educativo Arquidiocesano de Bogotá-SEAB de la Localidad Rafael Uribe Uribe (2016-2017), Hojas y Hablas, (16), 59-74. DOI:10.29151/hojasyhablas.n16a4
} 


\section{Introducción}

La educación en sus distintos niveles como fundamento del ciclo vital, desde la perspectiva de los discursos de desarrollo y calidad de vida, transversa los planes de diverso orden de la sociedad contemporánea, reconociéndose como un factor fundamental que aporta al bienestar social, si bien no es el único, por lo cual los recursos destinados para ella se asumen como una inversión para la superación de la desigualdad social, procurando para las personas el acceso a oportunidades y libertades ( Sen, 1999).

De manera paralela a las políticas de Estado sobre educación, resulta significativo el papel de diversas organizaciones de la sociedad civil que participan en el campo educativo. En el contexto latinoamericano y nacional, un actor presente es la Iglesia Católica, a través de instituciones que profesan en su misionalidad y visión, de la mano de la evangelización y como herramienta para su cumplimiento, el ejercicio de la labor educativa básica, media, pre-gradual y post-gradual.

Se observa en los estudios revisados el predominio de temas alrededor de la institución católica en procesos de evangelización y su rol en el marco de los cambios sucedidos en el campo educativo a partir de la secularización donde se profundiza en las relaciones entre el Estado, la Iglesia y la educación y el impacto de la religión alrededor de la inequidad social en la perpetuación o transformación de las estructuras sociales en América Latina (Hornich, D., Evangelista, F. \& Miranda, A. (2016).

Si bien se reconoce en América Latina la escasez de estudios sobre la educación católica (Torrendell, 2010), se destaca la importancia entre otros, de cuatro aspectos de esta institución alrededor de los valores en la escuela: sobre quién enseña, cómo enseña, en qué enseña y para qué enseña y las posibilidades que ofrece a sus estudiantes para ser más participativos, democráticos, tolerantes, abiertos a la discusión desde un pensamiento crítico del mundo social (Aparecida, 2007), así como las tensiones entre las legislaciones vigentes y los derechos de los padres para decidir sobre la formación moral y religiosa de sus hijos.

Se cuestiona la educación que se asume cada vez más desde una perspectiva utilitarista con relación a las reformas del campo educativo que buscan responder a las demandas en el marco del cambio global, las cuales se centran en "la adquisición de conocimientos y habilidades, y denotan un claro reduccionismo antropológico, ya que conciben la educación preponderantemente en función de la producción, la competitividad y el mercado" (Aparecida, 2007, p. 179). Desde ese escenario se afirma que el fin de la escuela es el ser un "lugar de formación integral mediante la asimilación sistemática y crítica de la cultura" (Aparecida, 2007, p. 179). En la producción sobre la educación católica se destacan los aportes de sus intelectuales en la literatura pedagógica, así como en la creación de centros de formación pedagógica (Torrendell, 2010).

Los estudios, siguiendo a Torrendell (2010) registran los dos procesos articulados propios de la modernidad que han modificado las relaciones de la Iglesia Católica con la educación; por una parte, la citada secularización y por otra, cierta fragmentación al interior de la institución, "que limita la comunión eclesial y que no se reduce sólo a un proceso sociológico, sino que afecta al corazón mismo de nuestra religión" (p. 302).

Si bien, en el campo educativo es indiscutible la importancia de la religión, no se cuenta con un 
estudio de impacto sobre esta labor que inicia desde la llegada de españoles y portugueses con la colonización y evangelización de América hasta la actualidad. (Torrendell, 2010) distingue tres momentos de la historia de la religión y el campo educativo específicos para América Latina; un primer momento del monopolio de la educación en manos de la Iglesia Católica (siglos XVI a XIX), un segundo a partir de las tensiones que surgen entre el Estado y la Iglesia Católica después de la independencia de los países latinoamericanos (entre 1810 y finales del siglo XIX) y un tercero que corresponde a "la consolidación del Estado Educador y el crecimiento paralelo de la educación católica" (finales del siglo XIX y década de los años 70 del siglo XX) (Torrendel, 2010, p. 286).

En el país, según el Ministerio de Educación Nacional, se encuentran registrados 22.350 colegios (2017). En Bogotá, 151 de ellos son religiosos católicos. En la localidad Rafael Uribe Uribe, 26 instituciones educativas son oficiales, 86 instituciones educativas son no oficiales y 12 de ellas son católicas, entre particulares, parroquiales y diocesanas, donde siete de ellas (una diocesana y seis parroquiales) pertenecen al SEAB (Dirección Local de Educación Rafael Uribe Uribe, 2015).

El SEAB; Sistema Educativo de la Arquidiócesis de Bogotá, está integrado por 19 colegios de distintas localidades, operados por fundaciones y parroquias y una institución de educación superior; Fundación Universitaria Monserrate. El SEAB pretende unificar tres aspectos fundamentales: el proceso evangelizador, el proceso pedagógico y el proceso administrativo [...] de acuerdo a unos criterios y políticas arquidiocesanas que la hagan parte de un sistema más eficaz en la prestación del servicio formativo (SEAB, 2015), donde se sigan los lineamientos de la Iglesia en relación con la escuela católica, en términos de:

Su finalidad y especificidad [así como en] las directrices del nuevo Plan de Evangelización [y] la concreción de un modelo pedagógico que responda a un nivel académico alto y a la vez sea un espacio en el que el proceso evangelizador se haga realidad en todos los espacios de la vida escolar (SEAB, 2015, pág. s.p.).

El lema del SEAB: Formar excelentes seres humanos, auténticos cristianos y verdaderos servidores de la sociedad. (SEAB, 2015), sintetiza sus directrices.

Según datos de la Arquidiócesis, para el año 2013 (creación) los colegios del SEAB contaban con 16.295 estudiantes.

La localidad 18 Rafael Uribe Uribe, donde se adelantó el estudio, está ubicada en la parte suroriental de Bogotá donde se levantaron antiguas haciendas y hoy se encuentran zonas residenciales y comerciales minoristas. Cuenta con 423.000 habitantes y 114 barrios (Secretaria Distrital de Salud, 2014). Su historia reciente corresponde a dinámicas de urbanización producto de procesos populares con la presencia de barrios legales, planificados e ilegales. Desde el inicio del siglo XX, se han sucedido oleadas de migración producto de la violencia sociopolítica y la expulsión económica, especialmente de población proveniente del campo colombiano. Estas dinámicas migratorias han dado lugar a procesos de organización comunitaria, donde la Iglesia Católica ha estado presente en los desarrollos barriales de la localidad.

La necesidad sentida de servicios para sus pobladores incluye las demandas en educación. Según estudios de diagnósticos locales como los adelantados por la Secretaría Distrital de Salud 
(1997-1998, 2003-2005), la Encuesta de Calidad de Vida y la Encuesta de Bogotá Cómo Vamos (2015), la educación ha venido presentando importantes avances en la localidad. De ahí la importancia de profundizar en el impacto social que visibiliza los alcances del quehacer educativo de la Iglesia Católica en la población para potenciar y generar estrategias que redunden en el bienestar social de sus usuarios, familias y comunidad, por lo cual se define el objetivo general del estudio:

Comprender el impacto social de los siete establecimientos educativos de la Localidad Rafael Uribe Uribe que hacen parte del SEAB, en sus estudiantes, egresados, familias y comunidad para potenciar y/ o proponer estrategias y líneas de acción.

Donde existe una necesidad sentida por investigar cómo impacta socialmente la escuela católica la comunidad académica y la comunidad externa.

\section{Marco teórico-conceptual}

En la amplia literatura sobre el impacto social, Liberta (2007) recoge desde diversos autores las concepciones sobre impacto; como la impronta o huella que se produce por una acción, el impacto social; como las consecuencias que se observan en el campo social a través de un proyecto y la evaluación de impacto social; como una forma de medición de los cambios que se producen en algún ámbito social. Si bien el estudio del impacto social articula aspectos como la pertinencia, eficacia, eficiencia, va más allá de ellos porque comprende cambios, logros, efectos previstos e imprevistos, los cuales son confrontados con las expectativas de los usuarios internos y externos, la misión y visión de la organización y el contexto donde se estudia, describiendo las consecuencias económicas, técnicas, sociales, culturales, políticas y ecológicas, entre otras, que se pueden presentar.

Es así como el impacto social es resultado de procesos y prácticas sociales que llevan a cabo las personas y organizaciones de diverso orden, que involucran productos alcanzados, pero es más que los productos que evidencia. El impacto social tiende a permanecer en el tiempo a mediano y largo plazo e implica conocer los efectos de un proceso con relación a unas metas propuestas, donde se da cuenta de las estrategias implementadas. Para Pichardo, el impacto social se enfoca "en los efectos deseados en el contexto social en que se inscriben las políticas, programas y proyectos, que se reflejan en cambios o variaciones en las variables, que pueden ser imputables a la intervención de aquellos" (Pichardo, 1995, p. 48).

La educación se aprecia como actividad, como relación con un enlace de transmisión de conocimiento y con un resultado, a través de una tecnología del proceso educativo, que de acuerdo a un sistema escolar da cuenta del campo educativo y su intersección con los otros campos sociales. Desde el constructivismo estructuralista propuesto por Bourdieu, se observa el campo de la educación básica y media como parte del sistema escolar junto con la educación superior, como el lugar donde se forman las personas, aportando a la configuración en su forma de pensar, de actuar y de ser en sociedad, es decir, se delinea su habitus y se pone en juego el capital humano como resultado del proceso de interiorización del conocimiento, donde el reconocimiento por ese conocimiento en las evaluaciones del estudiante y de la institución, otorga un estatus de acuerdo a un capital simbólico, que caracteriza el campo educativo (2011).

Bourdieu (2001) da cuenta en uno de sus primeros estudios de una lectura crítica del campo 
educativo, en relación con las posibilidades que ofrece la educación para el ascenso social, a través del capital humano que adquieren sus beneficiarios. La posición en el campo social y las posibilidades de acceder a los distintos capitales inciden en el impacto social de las instituciones educativas y de sus educandos, que no se puede desconocer para comprender los alcances de dicho impacto, asociado a las condiciones socioeconómicas y culturales de sus familias y del lugar que ocupan en el campo social, que se relaciona con sus posibilidades de acceso a los distintos capitales (culturales, sociales, económicos, simbólicos, entre otros).

De esta manera, la escuela es una institución fundamental para la adquisición de capital humano y social que no se puede desconocer. El proceso de enseñanza-aprendizaje influye en las diferentes dimensiones y comportamientos del ser humano en la sociedad y en sus posibilidades para el ingreso a la educación superior.

A su vez, el capital humano obtenido se traduce en otros como el económico, a lo largo de la vida de los agentes que, junto con la familia, dan cuenta en parte sobre cómo se observa el mundo social y la reproducción de las estructuras sociales que propicia. Se cuestiona hasta dónde la educación puede permitir el ascenso social sin desconocer los mecanismos sociales que operan en relación con el origen social y el lugar que se ocupa en el campo social (Bourdieu, 2011).

De igual manera, la teoría del capital social, siguiendo a Durston (2000), permite rescatar aportes anteriores de teóricos como R. Putnam, donde se definen los elementos que lo conforman, como lo son la cooperación, la solidaridad y la confianza, donde la escuela se constituye en un espacio de formación y entrenamiento para la ampliación del capital social, en términos personales e institucionales, entendiendo el capital social como el conjunto de vínculos que surgen a partir de las interacciones sociales que dan cuenta del tejido social. En las formas como se asumen estas interacciones, se va definiendo el tejido social y las posibilidades de conformar diversas redes.

\section{Metodología}

El estudio se llevó a cabo entre el 2016 y primer semestre de 2017, con 7 colegios de la localidad Rafael Uribe Uribe de la ciudad de Bogotá; Instituto San Pablo Apóstol, Colegio Parroquial San Juan Bautista de La Salle, Colegio Parroquial San Ignacio de Loyola, Colegio Parroquial Nuestra Señora, Colegio Parroquial San Luis Gonzaga, Colegio Parroquial Inmaculado Corazón de María y Colegio Parroquial San José. El tipo de investigación corresponde a una de impacto social no recurrente (Pichardo, 1998). El enfoque es mixto (Hernández, Baptista \& Fernández, 2010), se trabajaron las técnicas de entrevista abierta y semiestructurada (Bonilla \& Rodríguez, 2005) con rectores, coordinadores académicos, cuyos resultados se triangularon con encuestas realizadas a docentes y estudiantes, como se indica en la tabla 1. 
Tabla 1. Miembros de las comunidades académicas entrevistados y encuestados por colegio

\begin{tabular}{|c|c|c|c|c|c|c|c|c|}
\hline \multirow[t]{2}{*}{ COLEGIO } & \multicolumn{4}{|c|}{ ENTREVISTADOS } & \multicolumn{4}{|c|}{ ENCUESTADOS } \\
\hline & Rector & $\begin{array}{l}\text { Vicerrector } \\
\text { Coordinador }\end{array}$ & Docentes & $\begin{array}{l}\text { Miembros } \\
\text { comunidad }\end{array}$ & Estudiantes & $\begin{array}{c}\text { Padres } \\
\text { de } \\
\text { familia }\end{array}$ & Docentes & Egresados \\
\hline L.P. SAN JOSE & 1 & 2 & 1 & 4 & 85 & 54 & 2 & 3 \\
\hline $\begin{array}{l}\text { C.P. SAN LUIS } \\
\text { GONZAGA }\end{array}$ & 1 & 1 & 0 & 3 & 280 & 32 & 4 & 4 \\
\hline $\begin{array}{l}\text { C.P. } \\
\text { INCODEMAR }\end{array}$ & 1 & 1 & 0 & 1 & 297 & 44 & 42 & 0 \\
\hline $\begin{array}{l}\text { C.P. NUESTRA } \\
\text { SEÑORA }\end{array}$ & 1 & 1 & 0 & 4 & 97 & 14 & 7 & 5 \\
\hline $\begin{array}{l}\text { C.P. SAN } \\
\text { IGNACIO }\end{array}$ & 1 & 1 & 8 & 5 & 206 & 29 & 0 & 6 \\
\hline C.P. LA SALLE & 1 & 1 & 0 & 3 & 132 & 14 & 21 & 6 \\
\hline $\begin{array}{l}\text { INSTITUTO SAN } \\
\text { PABLO }\end{array}$ & 1 & 1 & 2 & 1 & 111 & 4 & 2 & 2 \\
\hline TOTAL & 7 & 8 & 11 & 21 & 1208 & 191 & 78 & 26 \\
\hline GRAN TOTAL & & & & 155 & & & & \\
\hline
\end{tabular}

Fuente: Equipo de Trabajo, 2017

\section{Hallazgos}

Sobre los resultados se aprecian similitudes, divergencias y tensiones alrededor de las categorías planteadas en los colegios estudiados en relación con el origen, desarrollo, discurso y cultura organizacional, comunidad académica, comunidad externa y redes sociales, de acuerdo a tres dimensiones; histórica, cultural y social, que se tienen en cuenta para comprender el impacto social de los colegios.

\section{Dimensión Histórica}

Sobre la dimensión histórica, a excepción del Instituto San Pablo Apóstol-ISPA, son colegios parroquiales donde la parroquia juega un papel dinamizador en la organización social con un impacto social positivo desde sus inicios. Su origen responde a la ausencia del Estado en sectores populares donde entre la urbanización legal e ilegal, las parroquias se constituyen en voceras, mediadoras y cogestoras con las comunidades para proveer el derecho a la educación a través de la construcción de los colegios. 
A su vez, los colegios que inician con primaria, van extendiendo sus servicios a medida que se adecua la infraestructura física para lograr cubrir entre los años 70 y 80 la prestación del servicio de educación básica y media y en la actualidad continúan adaptando sus instalaciones de acuerdo a los requerimientos de sus comunidades educativas.

Un segundo aspecto en términos históricos se encuentra a partir de la administración de Lucho Garzón (2004-2007) con la construcción y puesta en marcha de los mega colegios con educación gratuita, por lo cual disminuyen las matrículas en los colegios de la Arquidiócesis, generando crisis económicas en algunas instituciones y obligando en ciertos casos a cerrar alguna de las sedes. Un tercer aspecto y en un tiempo más reciente se relaciona con la crisis social que ha permeado la escuela, incluyendo los grandes colegios, exponiendo a los niños e incluso docentes a problemáticas de los sectores donde funcionan como son la inseguridad, el microtráfico y consumo de SPA, la presencia de pandillas, con formas de violencia ya presentes en la escuela con otras emergentes, que han reorientado la demanda hacia los colegios parroquiales nuevamente, lo cual incrementa el número de estudiantes. Al respecto se tiene que, según los estudiantes, padres de familia y docentes, los tres problemas más relevantes alrededor de los colegios del SEAB de la Localidad Rafael Uribe, en adelante LRUU, son el expendio y consumo de SPA (41\%), el hurto en espacios públicos (34\%) y la falta de presencia de la policía (25\%).

La escuela católica se asume como una burbuja protectora en un medio que se observa más amenazante para los niños. Las horas de entrada y salida, así como las rutas de acceso a los colegios resultan ser escenarios de alerta para toda la comunidad académica, que trata de aliarse con la policía del cuadrante, variando su presencia de una institución a otra.

Este aspecto no elude que al interior de las instituciones se observen algunas de estas problemáticas, pero manifiestan los integrantes de la comunidad académica que ante cualquier indicio se hace seguimiento y verificación para evitar o superar situaciones que afecten a los niños.

La sinergia de la triada parroquiacomunidad-colegio impulsa el desarrollo de las instituciones. De manera excepcional, el Instituto San Pablo Apóstol (ISPA), no se fundó articulado a una parroquia específica, si bien es producto del liderazgo espiritual católico. Otro aspecto que lo diferencia con los seis colegios restantes es la gratuidad a través de un plan de auto sostenibilidad exitosa, con la creación de empresas industriales que administra y con las cuales se financia la educación de los niños. Los demás colegios requieren para su funcionamiento ingresos a través del cobro de matrículas y pensiones.

La sede del ISPA ubicada en la localidad corresponde a educación media y su ingreso está mediado por las calidades académicas de estudiantes de ingresos bajos y medios, sustentado en la corresponsabilidad; el colegio se esfuerza en ofrecer una educación de alta calidad para que el estudiante continúe obteniendo un alto rendimiento académico. De igual manera, el colegio cuenta con un reconocimiento por parte de los colegios de la Arquidiócesis y del campo educativo en general, al ocupar lugares destacados en las pruebas de Estado en la ciudad.

\section{Dimensión Cultural}

El discurso cristiano católico que sustenta la Iglesia Católica incide en la configuración, ubicación y reconocimiento en el campo educativo de sus instituciones. La cultura organizacional presente 
en la Arquidiócesis de Bogotá es una categoría desde la cual se puede observar cómo se posibilitan las aspiraciones institucionales. Dos conceptos articulados entran aquí en el análisis; la organización y la cultura. La organización se entiende como una expresión cultural, más que una entidad que cuenta con una cultura, la cual resulta ser el producto de las interacciones sociales donde se evidencian las prácticas sociales.

Tanto la comunidad externa, como la misma comunidad académica aprecian la formación en valores ante las problemáticas del contexto donde se ubican los colegios parroquiales. La evangelización implica la formación en valores cristianos católicos que trasversa el Proyecto Educativo Institucional, guía las estrategias educativas, de tal manera que el perfil administrativo, docente y del estudiante se fundamenta en dichos valores, que se articula con el plan de estudios y los métodos pedagógicos que se llevan a cabo en su cotidianidad, buscando ser cada vez más integral, al aunar esfuerzos para formar excelentes seres humanos, auténticos cristianos y verdaderos servidores de la sociedad, como propone la Arquidiócesis de Bogotá. Por lo cual las instituciones consideran que el docente como persona y profesional de la educación se fortalezca, actualice y acompañe, evidenciando una mirada sistémica de las organizaciones educativas que integran el discurso en la acción para lograr dicho perfil.

Los estudiantes y padres de familia tienen una percepción positiva de los docentes, resaltan la forma de ser y aspectos que tienen que ver con la manera como ejercen su función docente como se observa en la gráfica 1.

Gráfica 1. Aspectos que le gustan de los profesores de los colegios del SEAB de la LRUU, a los padres y estudiantes er

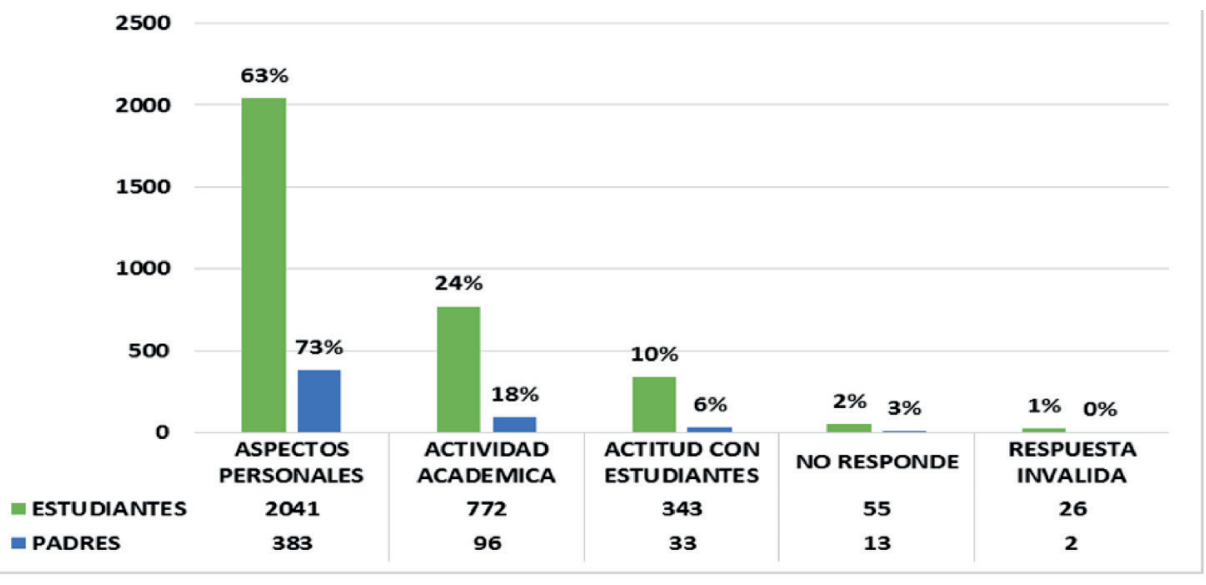

Fuente: Equipo de Trabajo, 2017

CCon relación al perfil de los estudiantes según los docentes encuestados se busca formar un excelente ser humano (42\%), con capacidad de aprender (23\%) y auténticos cristianos (17\%).

Las familias de los niños que estudian en estas instituciones se ubican generalmente en estratos tres y dos y en menor proporción en uno. De acuerdo a las entrevistas adelantadas, directivos y docentes destacan que el perfil de las familias de los estudiantes ha venido cambiando como referente a su vez de los cambios sociales en el mundo contemporáneo. Por ejemplo, en los últimos años se observa un incremento de padres con educación superior, pero prevalecen aquellos con un menor nivel de educación, aunque no 
se cuenta con estadísticas al respecto. Suelen habitar el sector donde se ubica la institución con algunas excepciones. Se observan casos recurrentes de egresados que matriculan a sus hijos en los colegios donde terminaron su bachillerato e incluso pueden alcanzar una tercera generación.

En relación con las familias, el discurso cristiano católico se encuentra en tensión en la cotidianidad, debido a los cambios sociales y culturales en su configuración y permanencia. Si bien se mantiene presente la tipología de la familia nuclear, reconocen directivos, coordinadores y docentes que cada vez es mayor el porcentaje de separaciones y divorcios, de madres y en menor proporción, padres cabeza de hogar, de niños al cuidado de abuelos y parientes, de la ausencia de alguno de los padres, de la demanda del sistema laboral y sobrecarga en la familia, donde ambos padres si están presentes deben salir a trabajar en muchas ocasiones; en estos casos se genera dolor e inestabilidad emocional en los niños que se traslada a su proceso de crecimiento en todas su áreas al crecer en soledad en el espacio más íntimo. Las estrategias alrededor de tiempos extracurriculares, el acompañamiento de psicólogo y/ o trabajador social y el papel del docente que brinda apoyo emocional propician un mejor acompañamiento, pero no provee como tal los soportes que debe ofrecer la familia.

De igual forma se presentan dificultades en el cumplimiento de los pagos de matrículas y pensiones por parte de algunos padres de familia, quienes a veces se encuentran desempleados o por alguna otra razón se reducen sus ingresos, afectando a su vez las finanzas de las instituciones.

Sobre los aportes que según los estudiantes de los colegios obtienen, se indican los resultados en la siguiente tabla.

Tabla 2. Aportes a la formación que hacen los colegios del SEAB a sus estudiantes de la LRUU

\begin{tabular}{l|c|c|c|} 
Aportes & \multicolumn{2}{c}{ SI } & \multicolumn{1}{c}{ A través de } \\
\cline { 2 - 4 } & No. & $\%$ & LA FORMACION ACADÉMICA \\
\hline SOCIAL & 985 & 81 & LOS EVENTOS DEPORTIVOS \\
\hline DEPORTIVO & 962 & 80 & LAS DIFERENTES MODALIDADES ARTISTICAS
\end{tabular}

Fuente: Equipo de Trabajo, 2017

Con relación a los padres de familia, la encuesta señala que el $89 \%$ reconoce que los estudiantes se caracterizan por sus aspectos personales positivos referidos a las actitudes, valores, principios, características personales, que les permite ser y relacionarse positivamente con los demás. El 65\% expresa que lo anterior se debe a la influencia en la formación humana integral que brindan los colegios.

Sobre la percepción de los padres por parte de los docentes se observa que el 49\% expresa que los padres de familia se caracterizan por su calidad humana, aspecto que corrobora el $99 \%$ de los padres. De igual forma, el $18 \%$ de los docentes expresa que se presentan problemas de disfuncionalidad familiar con las mismas problemáticas ya expuestas en las entrevistas a directivos, coordinadores y docentes donde se reportan casos (no corresponde a la mayoría) de descuido, de padres ausentes e incumplimiento de la responsabilidad parental en el seguimiento educativo de sus hijos, si bien reportan todos contar con escuelas de padres, propiciar escenarios de encuentro y algunas dinámicas de seguimiento para concienciar 
sobre su rol en el progreso académico y bienestar de los niños.

El capital cultural como interiorización de saberes que adquieren los niños, se evidencia en las dos razones con mayor porcentaje que expresan, a través de las encuestas, los padres de familia para escoger los colegios del SEAB: calidad académica (46\%) y formación integral (32\%), como se presenta en la siguiente gráfica.

Gráfica 2. Razones por las cuales los padres escogieron los Colegios del SEAB de la LRUU para sus hijos

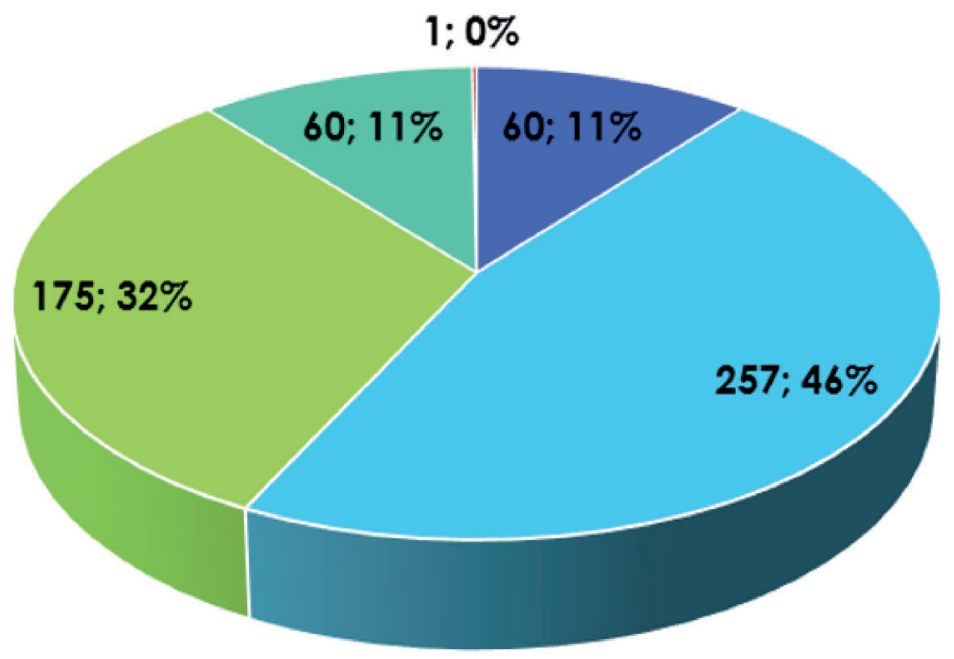

- LA UBICACIÓN

- LA CALIDAD ACADEMICA

- LA FORMACION INTEGRAL

- LA TRADICION

- NO RESPONDE

Sobre las apuestas pedagógicas, las instituciones de manera más o menos explícita apuntan a la promoción de una filosofía del cuidado que se incorpore en el habitus de los miembros de las organizaciones y sus estudiantes, que se haga presente en las decisiones y prácticas morales, donde pensar éticamente es pensar en los demás (Comins, 2009). El enseñar en comunidad la competencia para la solidaridad, para cuidar unos de otros, como un fundamento de la convivencia, contiene unas implicaciones sociales que superan el ámbito del campo educativo. Para los estudiantes, los colegios estimulan comportamientos sociales referidos a actitudes o comportamientos positivos que observan en la relación con los otros (21\%) y evidentes en expresiones como: "amigables, amables, ayudar a otros, tener una buena convivencia, ser misericordioso, compañerismo, sentido social, servicial, liderazgo, servidores de la sociedad, solidaridad, hermandad y generosidad entre otros" (Encuesta-Estudiante, 2016).

Este sentido de cuidado también es evidente en las familias en la medida que los padres expresan que el colegio les brinda un fortalecimiento familiar (31\%) referido a: la unión familiar, la confianza mutua, el compartir, la comunicación, el buen trato, la sana convivencia, el apoyo y la armonía familiar. (Encuesta-Padres, 2016).

En relación con el discurso pedagógico, los colegios siguen el enfoque constructivista y humanista con algunas singularidades, el énfasis en 6 de ellos es académico y en el caso del Colegio Parroquial Liceo San José es comercial y se asume como un valor agregado al brindar una herramienta para la vida. 
Para lograr una educación de calidad, se destaca el trabajo colaborativo de la comunidad académica para cumplir con los objetivos propuestos de manera eficaz. La implementación del Sistema de Gestión de Calidad en las organizaciones y su recertificación es una dinámica permanente de todos los colegios del SEAB de la LRUU.

Otro aspecto que caracteriza la organización en estas instituciones es la jerarquía eclesiástica, con la dirección de sacerdotes de la Arquidiócesis de Bogotá, con experiencia en el campo de la educación, conocedores del contexto y de las realidades de sus beneficiarios y familias y como líderes espirituales de las parroquias, lo cual les permite tener cercanía en ambos ámbitos; en el campo social a través de la interacción con familias residentes, líderes sociales, instituciones locales y distritales, y, en el campo de la educación, en cabeza de la institución educativa. Esta simbiosis permite potenciar estrategias tanto educativas como sociales, desde la perspectiva religiosa que enriquecen las dinámicas del campo educativo.

Los rectores, coordinadores y docentes entrevistados manifiestan un gran aprecio y satisfacción por sus egresados, quienes en algunos eventos como bazares y encuentros vuelven a visitar a la comunidad académica. Se reconocen algunos logros como obtención de becas en programas como Ser Pilo Paga y su ingreso a universidades públicas y privadas de prestigio, para cursos postgraduales en el extranjero y el reconocimiento de algunas trayectorias laborales exitosas.

En seis de los colegios encuestados no hay una sistematización de sus egresados a través de una base de datos actualizada que permita evidenciar de manera confiable el impacto social del colegio en ellos, sus familias y entorno. Solo el Colegio Parroquial Inmaculado Corazón de María cuenta con una, además de un grupo organizado donde eventualmente se llevan a cabo encuentros. Este es uno de los factores que impide una mayor visibilización del impacto social de los colegios.

\section{Dimensión social}

En relación con la comunidad externa; tanto el colegio como el contexto donde se ubican coinciden en señalar la presencia de las problemáticas ya citadas arriba, así como la presencia de habitante de calle, mal manejo de basuras y la peligrosidad de algunas vías para los niños que se movilizan a pie. En las entrevistas a miembros de las comunidades donde se ubican las instituciones, destacan el buen comportamiento de los estudiantes y la calidad de la educación que ofrecen (Entrevistas comunidad, 2016), sin embargo, observan las instituciones como escenarios para algunos privilegiados; para los entrevistados las matrículas resultan costosas, mientras que para los directivos de los colegios se hace un gran esfuerzo para reducir su valor al máximo a cambio de prestar una educación de calidad. Es una tensión presente también en padres de familia; con esfuerzo pagan la educación de sus hijos y de acuerdo a sus ingresos les resulta costosa si bien la aprecian.

Con relación a los referentes que guían la prueba de Estado para todos los colegios del país, se asumen de acuerdo a principios del mercado educativo, desde una perspectiva más de servicio que como derecho y donde todas las instituciones deben de manera obligatoria participar. Se encuentra aquí otra tensión sobre cómo no desdibujar la función cristiana y social de la educación que se imparte ante las demandas del mercado que se traduce en la educación por competencias y en el reconocimiento mediado por el puntaje que se obtenga y no necesariamente por la labor social de responder a las necesidades de la población, incluidos los factores de incidencia directa en el impacto social que puede propiciar una educación de calidad, la cual también se puede ver reflejada en los hogares de los estudiantes.

Al respecto los padres de familia expresan que la formación que imparten los colegios del SEAB a sus hijos los ha influenciado principalmente en aspectos como lo personal (65\%) referido a comportamientos, actitudes y valores y 
lo académico (23\%) referidos al rendimiento académico alto, el interés por aprender, la dedicación y esfuerzo que le ponen al aprendizaje entre otros. (Encuesta-Padres, 2016). Para los padres, la escuela católica, en el caso de estas instituciones propicia transformaciones favorables al interior de sus hogares en términos de bienestar, modificando sus formas de relacionarse y en el deseo de superación a través del conocimiento.

Los colegios según la anterior clasificación (es la que refieren) se ubican en superior y muy superior. Algunos de ellos han ocupado primeros lugares en Bogotá como es el caso del ISPA.

En las instituciones se encuentra una tensión adicional; al competir por un mayor estatus en la medida en que obtengan mejores puntajes como capital simbólico, con una mejor ubicación en el ranking, lo cual exige una mayor inversión, y de igual manera, cumplir una función social de proveer acceso a educación de calidad a bajos precios y para sectores más o menos vulnerables. Un agregado social que no suele medirse para evaluar a los colegios es el cambio socioeconómico que a mediano y largo plazo puede generar en los estudiantes, al concentrarse el ranking sólo en los puntajes. Por ejemplo, en relación con niños que de acuerdo a su formación académica corresponden a primeras generaciones que ingresan a la educación superior y las transformaciones que conllevan el contar con una formación técnica, tecnológica o profesional en el mercado laboral.

Es decir, no se evalúa cómo el capital humano que se obtiene se transforma en capital económico al permitirle a su beneficiario contar con mayores posibilidades de obtener un empleo con mejores condiciones. De igual manera, el capital se transforma también en social, aportando a un mayor bienestar.

En este orden de ideas se observa en los colegios estudiados la lucha por los capitales propios del campo educativo, de acuerdo a los lineamien- tos estatales en materia de educación, en una competencia para obtener unos resultados que los clasifique en el puntaje mayor y para lo cual definen sus planes de trabajo. Entre ellos, evitar en algunos casos, recibir estudiantes en grados superiores que afecten sus puntajes. Se tiende a seleccionar estudiantes que provienen de otras instituciones cercanas que cuenten con rendimiento académico alto, lo cual deja por fuera a aquellos que requieren también educación de calidad pero que de acuerdo a su record académico pueden desmejorar el nivel del colegio al presentar las pruebas de Estado.

Los lineamientos éticos de la política de educación compaginan en gran medida con el discurso católico, lo cual no exime de divergencias frente a otras ideologías en relación, por ejemplo, con valores asociados al sentido y expresión de la sexualidad, que en estas instituciones se aborda desde la concepción católica, haciendo énfasis en la dignidad humana y la vivencia de valores.

En este aspecto, los colegios toman distancia de propuestas educativas distritales y de algunas provenientes del campo de la salud. En el proceso de institucionalización, del establecimiento de las prácticas y de las relaciones sociales que se propician en las instituciones educativas, está presente en la propuesta educativa la filosofía del cuidado ya citada.

Sobre la opinión de los colegios acerca del SEAB a partir de su creación, entrevistados y encuestados coinciden en su importancia para acompañar el trabajo conjunto en relación con la reforma curricular, el proyecto de vida y favorecer espacios de encuentro de coordinadores académicos y de integración en actividades deportivas, a través de campeonatos inter-colegiados, en actividades académicas como olimpiadas de matemáticas, filosofía e inglés, encuentros de física y química, en el campo artístico con encuentros de danzas y bandas, actividades litúrgicas en distintas sedes, entre otros. En el caso de la semana anual del 
SEAB confluyen diferentes manifestaciones donde comparten escenarios algunos colegios.

La interacción comunitaria, institucional e interinstitucional aporta al sostenimiento de los colegios parroquiales. Con relación a la percepción de los estudiantes sobre el trabajo con la comunidad y otras instituciones no es tan visible como se quisiera; tan solo el $36 \%$ considera que el colegio realiza actividades con la comunidad barrial. El 51\% señala que cuenta con algunos escenarios de encuentro con otras instituciones donde realizan diferentes actividades como: Bingo bazar, juegos inter-colegiados, feria universitaria, festivales, foros y eventos formativos, olimpiadas de matemáticas y filosofía, salidas pedagógicas, debates, feria de bandas, presentaciones artísticas $<$ danzarte $>$ (Encuesta-Estudiante, 2016). Para los docentes los espacios de participación con otras instituciones se dan en la Semana SEAB (24\%), especialmente en actividades deportivas, con un 19\%. (Encuesta-Docentes, 2016).

Con otras instituciones las relaciones son más puntuales, como préstamo de las instalaciones de los colegios a instituciones locales y algunos eventos y campañas que se suman a los escenarios usuales del campo educativo en la localidad y el Distrito con las entidades rectoras del sistema educativo distrital y nacional.

Específicamente, destacan los convenios con el SENA del ISPA y el Colegio Parroquial San Luis Gonzaga, así como el trabajo en red del ISPA para avanzar con su proyecto industrial con el cual financia la educación de sus beneficiarios.

Con las comunidades del sector la intensidad y nivel de relaciones varía de una institución a otra. En algunos casos las observan lejanas y desconocen su trabajo, en otros casos aprecian que se ubiquen en su sector por las dinámicas urbanas que se generan alrededor de actividades como las comerciales y por el buen comportamiento que destacan de sus estudiantes y de las relaciones cordiales con sus directivos y personal, y, el acompañamiento que ofrecen para la solución de problemáticas comunes así como el reconocimiento de la población católica que hace parte de las parroquias articuladas a los colegios estudiados.

En la triangulación de la información coinciden docentes y estudiantes sobre una percepción positiva de la comunidad respecto al colegio; los estudiantes creen que la comunidad dice que es una institución académicamente buena y los docentes dicen que resulta favorable su presencia en el sector y que imparte una formación integral.

Tabla 3. Opinión que tiene la comunidad sobre los colegios del SEAB de la LRUU, según los estudiantes y docentes encuestados

\begin{tabular}{|l|c|c|c|c|}
\hline & \multicolumn{2}{|c|}{ ESTUDIANTES } & \multicolumn{3}{c|}{ DOCENTES } \\
\hline & No. & $\%$ & No. & $\%$ \\
\hline BUENO ACADEMICAMENTE & 409 & 34 & 22 & 20 \\
\hline BUENO EN EL SECTOR & 282 & 23 & 38 & 35 \\
\hline NO RESPONDE & 209 & $17 \%$ & 15 & 14 \\
\hline FORMACION INTEGRAL & 145 & 12 & 18 & 16 \\
\hline BUENOS ESTUDIANTES & 80 & 7 & 11 & 10 \\
\hline OPINION NEGATIVA & 74 & 6 & 5 & 5 \\
\hline NINGUNA & 9 & 0,7 & - & - \\
\hline
\end{tabular}




\section{Recomendaciones}

El trabajo en red como el SEAB resulta relevante para potenciar y visibilizar el impacto social de los colegios, por lo cual se recomienda contar con estrategias de seguimiento a egresados a través de una línea de base que tenga en cuenta en cada institución, sus singularidades para medir, reconocer y potenciar el impacto social que la Iglesia Católica desde la educación aporta a la sociedad, a través de sus egresados. De igual manera, crear nuevos escenarios de encuentro y de redes interinstitucionales que potencien las interacciones tradicionales entre la comunidad académica y la comunidad externa.

Se recomienda una estrategia de evaluación y retroalimentación a través de estudios que permitan conocer los alcances del proyecto de vida de los egresados, sus opiniones y sugerencias acerca de la calidad de la educación recibida. A partir del seguimiento a egresados, con una base de datos actualizada, se propone contar con una red donde la comunidad académica y sus egresados cuenten con canales de comunicación y encuentro, potenciando los vínculos e interacciones en términos de apoyo presente y futuro para la institución, estudiantes y egresados, que incluya voluntariado y servicio social, apoyo para egresados que buscan trabajo, feria de negocios, oferta de servicios, encuentros culturales, entre otros.

A partir de la investigación realizada, una de las propuestas que se plantea es que se trabaje en los colegios un enfoque desde la educación popular entendida como un proceso participativo y transformador, donde todos los actores reconozcan en el contexto las estructuras y factores que determinan sus vidas, ayudándoles a desarrollar estrategias, habilidades y técnicas para que puedan llevar a cabo una participación orientada a la transformación de la realidad, (Eizagirre) con el apoyo de otras organizaciones y el trabajo en red que posibiliten la participación de actores (educativos, culturales, religiosos, barriales), intercambiando experiencias y saberes. Con ello se busca desde la escuela e insertado en el SEAB, seguir trabajando por una sociedad más justa y humana.

Se busca seguir fortaleciendo la red de origen como lo es el SEAB, a través de encuentros de coordinadores, orientadores y directivas. A lo anterior se puede sumar la identificación conjunta de recursos comunitarios e institucionales existentes en los distintos sectores donde se ubican los colegios, para crear vínculos y/ o fortalecerlos, realizando acercamientos, generando conexiones y convenios que permitan visibilizar estas organizaciones así como compartir experiencias, conocimientos y servicios. Se recomienda seguir impulsando la participación de las familias en el ámbito escolar para alcanzar una educación de calidad y propender por una prevención integral en la vida de los estudiantes, lo cual contribuye a la atención y resolución de algunas problemáticas relacionadas con la violencia escolar, el bullying, la soledad de los niños y la mala utilización del tiempo libre de los mismos, por lo cual se recomienda contar con una escuela de padres con estrategias que permitan crear espacios para dotarlos de herramientas actualizadas, donde los mismos padres formen a sus pares en aspectos de desarrollo humano, conocimientos y habilidades para tener un mejor desempeño con sus hijos, promoviendo la convivencia escolar y familiar, alrededor de la amistad, la lealtad y la construcción de paz.

Permitir que los padres formen parte de esa diada de la educación popular como es la escuela comunidad escuela es plantear iniciativas que contribuyan a resolver las problemáticas anteriormente mencionadas, y propiciar espacios para que ellos ejerzan las funciones que les otorga la crianza de los hijos.

\section{Conclusiones}

Los colegios estudiados reflejan a lo largo de su historia el impacto social, como respuesta a las necesidades de las poblaciones para suplir el derecho a la educación de sus niños, ante la ausencia inicial del Estado y de ofrecer la gratuidad 
en el caso del Instituto San Pablo Apóstol, bajo el lineamiento para lograr educación de calidad.

Las comunidades académicas destacan dos aspectos comunes; la educación con la formación en valores para la construcción del proyecto de vida y la identificación de sus miembros en las comunidades externas, con una buena formación en valores que se evidencia en el comportamiento que destacan como distintivos de los colegios del $\mathrm{SEAB}$ en la localidad, en términos de respeto y sana convivencia.

El trabajo en red, especialmente a través del Sistema Educativo de la Arquidiócesis de Bogotá, SEAB, refleja el reto de los colegios para seguir fortaleciendo su trabajo a través del aprendizaje de experiencias y profundización en el reconocimiento de los contextos y de la educación frente a los cambios de diverso orden del mundo social, con el fin de potenciar, por una parte, los logros obtenidos, y por otra parte, generar nuevas dinámicas acordes con el discurso cristiano católico frente a las demandas del mercado educativo, en el contexto de la globalización y el utilitarismo impulsado por el modelo neoliberal, que lo distinga frente a la prevalencia de la racionalidad económica.

El trabajo con egresados se constituye en una oportunidad para seguir ampliando el trabajo en red y medir con mayor precisión el impacto social de los colegios, desde una red mayor como lo es el SEAB. El seguimiento permitirá precisar los aportes de la formación que brindan los colegios a los estudiantes y sobre cómo potencia sus habilidades sociales y cognitivas para acceder con sus egresados, a diferentes campos del saber, laborales, económicos, culturales, sociales y políticos, bajo la impronta de la ética del cuidado del otro y de sí mismo.

\section{Referencias}

Bonilla, E., \& Rodriguez, P. (2005). Más allá del dilema de los métodos. La investigación en ciencias sociales. Bogotá: Norma.

Bourdieu, P. (2011). Capital cultural, escuela y espacio social. Mexico: Siglo Veintiuno editores.

Bourdieu, P., \& Passeron, J. (2001). La reproducción. Elementos para una teoria del sistema de enseñanza. Madrid: Editorial Popular.

Comins, I. (2009). Filosofía del cuidar. Una propuesta coeducativa para la paz. Madrid: Editorial Icaria.

Concejo de Bogotá (2015). Encuesta Bogotá Cómo Vamos. Recuperado 19 de abril de 2017 http://www.bogotacomovamos.org/ documentos/encuesta-de-percepcion-ciudadana-2015/

Consejo Episcopal Latinoamericano, CELAM, (2007). Documento Conclusivo. V Conferencia del Episcopado Latinoamericano y del Caribe. Aparecida, 13-31 de mayo. Bogotá: San Pablo. Recuperado de http:// www.vidanuevadigital.com/wp-content/ uploads/2013/04/Documento_Conclusivo_ Aparecida.pdf.

Departamento Nacional de Estadística (DANE) (2017). Encuesta Nacional de Calidad de Vida. Recuperado https://www.dane.gov. co/index.php/estadisticas-por-tema/salud/ calidad-de-vida-ecv/encuesta-nacional-decalidad-de-vida-ecv-2017

Durston, J. (2000). ¿Qué es el capital social comunitario? Santiago de Chile: Serie politicas sociales CEPAL-NACIONES UNIDAS.

Eizagirre, M. (s.f.). Educación Popular. (U. e. Vasco, Ed.) Recuperado el 2017, de Diccionario de acción humanitaria y cooperación al desarrollo: http://www.dicc.hegoa.ehu.es/ listar/mostrar/83

Hernández-Sampieri, R., Fernández-Collado, C., \& Baptista, P. (2010). Metodología de la investigación. Bogotá: MacGraw Hill.

Hornich, D., Evangelista, F. \& Carlos Miranda, 
A. (2016). Estado, religião e educação do cidadão. Acta Scientarium: Education, 38(2), 143-153. DOI:10.425/actascieduc.-v3 $8 \mathrm{i} 2.26758$

.Liberta, B. (2007). Impacto, impacto social y evaluación de impacto. Recuperado el 8 de Febrero de 2016, de Acimed: http://bvs.sld.cu/ revistas/aci/vol15_3_07/aci08307.htm

Minsiterio de Educación Nacional (2017). Datos Abiertos MEN. Recuperado el 5 de Noviembre de 2018 https://www.mineducacion.gov. co/1759/w3-propertyvalue-57277.html

Pichardo, A. (1997). Evaluación del impacto social: el valor de lo humano ante la crisis y el ajuste. Buenos Aires Argentina: Lumen Hvmanitas.

SEAB. (2015). Sistema Educativo de la Arquidiócesis de Bogotá. Recuperado el 4 de Febrero de 2016, de Justificacion y proceso de construcción: http://arquibogota.org.co/ es/noticias/category/sistema-educativo-dela-arquidiocesis-de-bogota---seab.htm Secretaria Distrital de Salud. (2014). Diagnóstico Local 2014. Obtenido de E.S.E. Hospital Rafael Uribe Uribe: http://www.saludcapital.gov.co/DSP/Diagnsticos\%20distritales\%20y\%20locales/Local/2014/18_DX\%20 LOCAL\%20RUU\%202014_11042016.pdf

Salud Capital. (2005). Diagnósticos locales con participación social Rafael Uribe Uribe Localidad 18. Obtenido de Colección Diagnósticos Locales de Salud con Participación Social: http://www.saludcapital. gov.co/sitios/VigilanciaSaludPublica/Diagnosticos\%20Locales/18-RAFAEL\%20 URIBE\%20URIBE.pdf

Sen, A, (1999). Desarrollo y Libertad. Barcelona: Editorial Planeta.

Torrendell, C. H. (2010). Desafíos de Fidelidad y Caridad en la Educación Católica Sudamericana. Humanitas (07172168), 15(58), 284301. 